\title{
Adult-Onset Orbital Sinus Pericranii with T2 Hyperintensity Lesion: A Case Report
}

\author{
Toshiya Osanai Kiyohiro Houkin \\ Department of Neurosurgery, Faculty of Medicine and Graduate School of Medicine, \\ Hokkaido University, Sapporo, Japan
}

\section{Keywords}

Sinus pericranii · T2 hyperintensity lesion · Adult · Vascular malformations

\begin{abstract}
Sinus pericranii is a rare vascular anomaly, and most cases occur in children and develop at the midline. In previous reports of sinus pericranii, T2 hyperintensity lesion has not been regarded as a common sequela. We report an extremely rare case of orbital sinus pericranii with associated $\mathrm{T} 2$ hyperintensity lesion. A 50-year-old man was admitted to our hospital with a history of right upper eyelid swelling that had been present for several years. Computed tomography, magnetic resonance imaging, and digital subtraction angiography demonstrated a connection between the lesion and normal cerebral venous system. Thus, we diagnosed the lesion as a sinus pericranii despite its atypical features. We elected to observe the patient, and the lesion had remained the same size without any adverse events, such as hemorrhage, occurring throughout the 5-year follow-up. An atypical sinus pericranii should be considered in patients with a soft compressible swelling on the head, even if the lesion is located off the midline.




\section{Case Reports in Neurology}

Osanai and Houkin: Adult-Onset Orbital Sinus Pericranii

\section{Introduction}

Sinus pericranii is a rare vascular lesion characterized by a venous communication between the intracranial dural sinus and extracranial venous system through the diploe of the skull. The anomaly is usually diagnosed during childhood; can be congenital, spontaneous, or traumatic in origin; and typically involves a single, fluctuant, nonpulsatile vascular swelling. The defect varies in size with changes in intracranial pressure, such as that which occurs during the Valsalva maneuver. The lesion is typically located at the midline adjacent to the superior sinus. Although a few cases of sinus pericranii located outside the midline have been described, these are extremely rare [1]. Previous reports have not described sinus pericranii accompanied by T2 hyperintensity lesion. We encountered a case of sinus pericranii located in the orbit that was associated with T2 hyperintensity lesion in an adult. Herein, we present this extremely rare case and review the pertinent literature.

\section{Case Report}

The study was approved by the Institutional Review Board of our Institute, and all patients provided written informed consent. A 50-year-old man was admitted to our hospital with right upper eyelid swelling that had been present for several years. He had experienced no head or birth trauma and had no other relevant medical history. He was first examined by plastic surgeons and diagnosed with a venous malformation. Sclerotherapy treatment was planned. However, as a precaution, our team was consulted to evaluate the lesion for a connection with the intracranial vasculature.

Our examination of the patient revealed no neurological deficits, but he had a soft nonfluctuant mass in the right upper eyelid that increased in size during the Valsalva maneuver and diminished with compression. Contrast-enhanced computed tomography (CT) showed a bony defect in the superior wall of the right orbit and an isodense mass lesion in the right orbit that did not enhance during the early phase after injection with contrast medium (Fig. 1). Magnetic resonance imaging (MRI) was also performed and indicated that the mass was hypointense on T1-weighted imaging and hyperintense on T2-weighted imaging. Additionally, T2-weighted imaging displayed hyperintense parenchyma near the lesion. Gadoliniumenhanced MRI demonstrated that the mass was a heterogeneous enhanced legion (Fig. 2). Digital subtraction angiography (DSA) demonstrated no abnormal vessels or other lesions in the arterial or capillary phase, but a venous lake was seen in the orbit during the venous phase. The venous lake received blood flow from the cortical vein of the frontal lobe. The vein draining the orbit could be clearly seen, but we could not determine whether this vein was intracranial (Fig. 3). Thus, we examined both DSA and MRI images and concluded that the orbital flow was draining to the extracranial temporal vein.

We diagnosed the patient with sinus pericranii based on these findings. After confirming the connection between the cerebral vein and the lesion, a plan for plastic surgical sclerotherapy was abandoned, and we used a conservative, outpatient, observational approach. The patient underwent follow-up every 6 months in the outpatient Department of Neurosurgery. At that time, MRI indicated that the size of the lesion had not changed, and there were no adverse events, such as hemorrhage, over the course of 5 years. 


\section{Discussion}

Herein, we presented a case of adult-onset orbital sinus pericranii. The case had some notable features that are atypical of sinus pericranii. First, the lesion developed in an adult of 50 years of age, and while this defect can occur at any age, most patients are $<30$ years old. Further, the patient had no history of trauma at birth or later in life, which was different from a typical observation in a congenital sinus pericranii. Conversely, acquired sinus pericranii appears to be caused by trauma, development of varicosities, or intracranial hypertension. However, in this case, the patient did not develop the condition due to any of these etiologies, which makes this case notable.

Second, the location of the defect was unusual in our case. In our patient, this lesion was located off the midline, even though most cases of sinus pericranii are located at the midline. Although some authors have reported this type of lesion in other areas, sinus pericranii within the orbit is very rare. Desai et al. [1] reported the case of a 16-year-old girl with sinus pericranii at the root of the nose with orbital extension. That case is similar to the one presented in this report.

The third difference between our case and previously reported cases was that the cerebral parenchyma adjacent to the sinus pericranii appeared hyperintense on T2-weighted MRI. To our knowledge, this feature has never been reported as a characteristic of sinus pericranii. We speculate that the thrombus caused a focal disturbance of the venous circulation, thereby resulting in T2 hyperintensity lesion which meant brain edema or encephalomalacia.

The occurrence of thrombosis is common in this condition because of the slow flow of blood within the sinus pericranii as well as the common occurrence of thrombi within venous malformations elsewhere in the body.

The diagnosis of sinus pericranii was based on the imaging characteristics of the lesion in this case. In short, the defect was distinguished from an arteriovenous shunt or tumor because DSA demonstrated communication between the intra- and extracranial venous systems without an arterial connection. Additionally, the lesion's change in size in accordance with patient position was strongly suggestive of a diagnosis of sinus pericranii. CT imaging showed the scalp defect and that the subcutaneous mass had a low density. The mass lesion showed homogeneous enhancement in the late phase. DSA revealed a connection between the intracranial vein and mass.

In this case, the accurate diagnosis was crucial because a sclerotherapy for sinus pericranii could have been dangerous. If the sclerosant material ran into the intracranial venous sinus, it could result in occlusion of the venous sinus, which may be fatal. Thus, we determined that our observation was appropriate. Many reports have described sinus pericranii as having a benign course, while Vinas et al. [2] reported that the lesion typically shows a stable or moderately increasing size. Historically, sinus pericranii has a low risk of bleeding, and observation is recommended in the absence of cosmetic concerns [3]. In this case, the asymptomatic nature of the condition, relatively mild cosmetic effects, and the functional role played by the malformation in venous drainage from the frontal lobe all contributed to our decision to observe the patient.

In conclusion, sinus pericranii with atypical features should be considered in patients with soft compressible swelling on the head, even when the lesion is located off the midline. 


\section{Case Reports in Neurology}

\section{Statement of Ethics}

The patient provided written informed consent.

\section{Disclosure Statement}

This study received no funding and the authors declare no conflicts of interest.

\section{References}

1 Desai K, Bhayani R, Goel A, Muzumdar D. Sinus pericranii in the frontal region: a case report. Neurol India. 2001 Sep;49(3):305-7.

2 Vinas FC, Valenzuela S, Zuleta A. Literature review: sinus pericranii. Neurol Res. 1994 Dec;16(6):471-4.

3 Gandolfo C, Krings T, Alvarez H, Ozanne A, Schaaf M, Baccin CE et al. Sinus pericranii: diagnostic and therapeutic considerations in 15 patients. Neuroradiology. 2007 Jun;49(6):505-14.
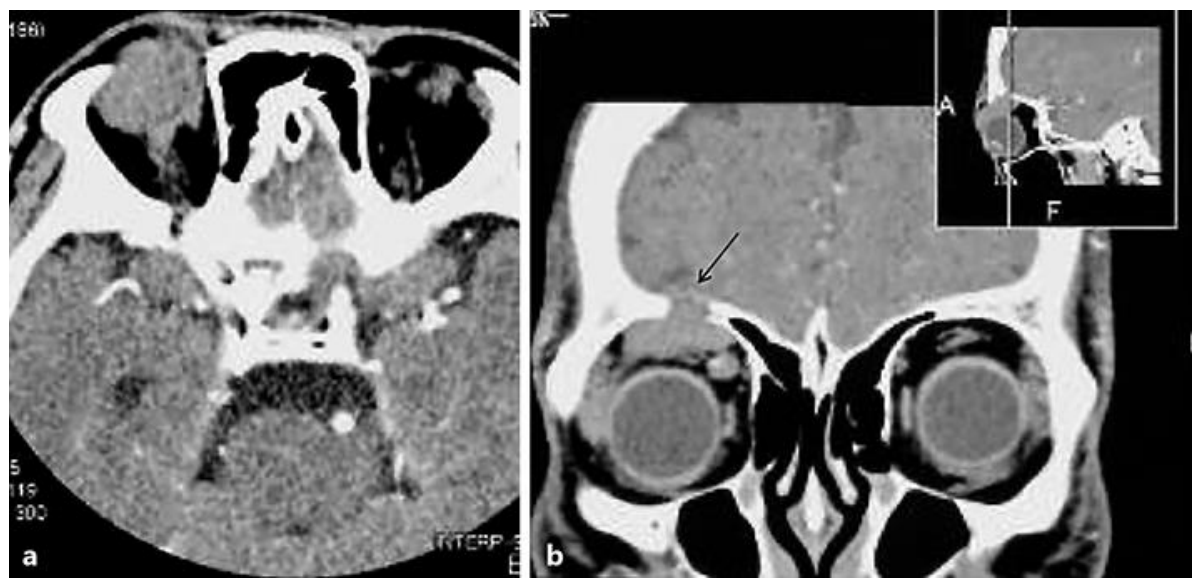

Fig. 1. Early-phase contrast-enhanced computed tomography scans show a nonenhanced mass in the right orbit and an orbital roof bone defect (arrow): axial view (a), coronal view (b). 


\section{Case Reports in Neurology}
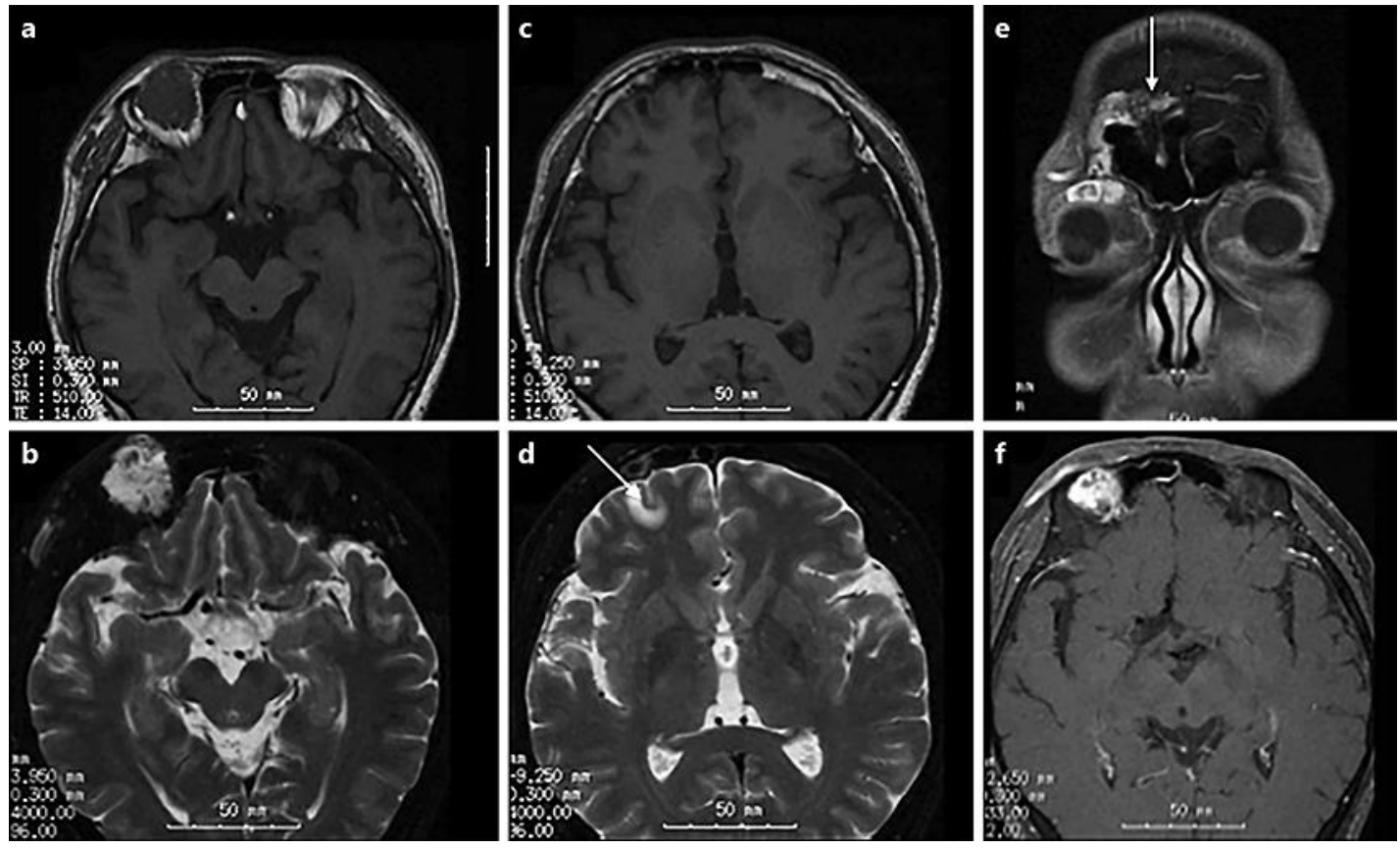

Fig. 2. Axial magnetic resonance images reveal a right orbital lesion that is of low intensity on T1 weighting (a) and high intensity on T2 weighting (b). The parenchyma near the sinus pericranii is of low intensity on T1 weighting (c) and high intensity on T2 weighting (arrow) (d). e, f The orbital mass lesion demonstrates gadolinium contrast enhancement and connects with the intracranial structure (arrow). 


\section{Case Reports in Neurology}

www.karger.com/crn

Osanai and Houkin: Adult-Onset Orbital Sinus Pericranii
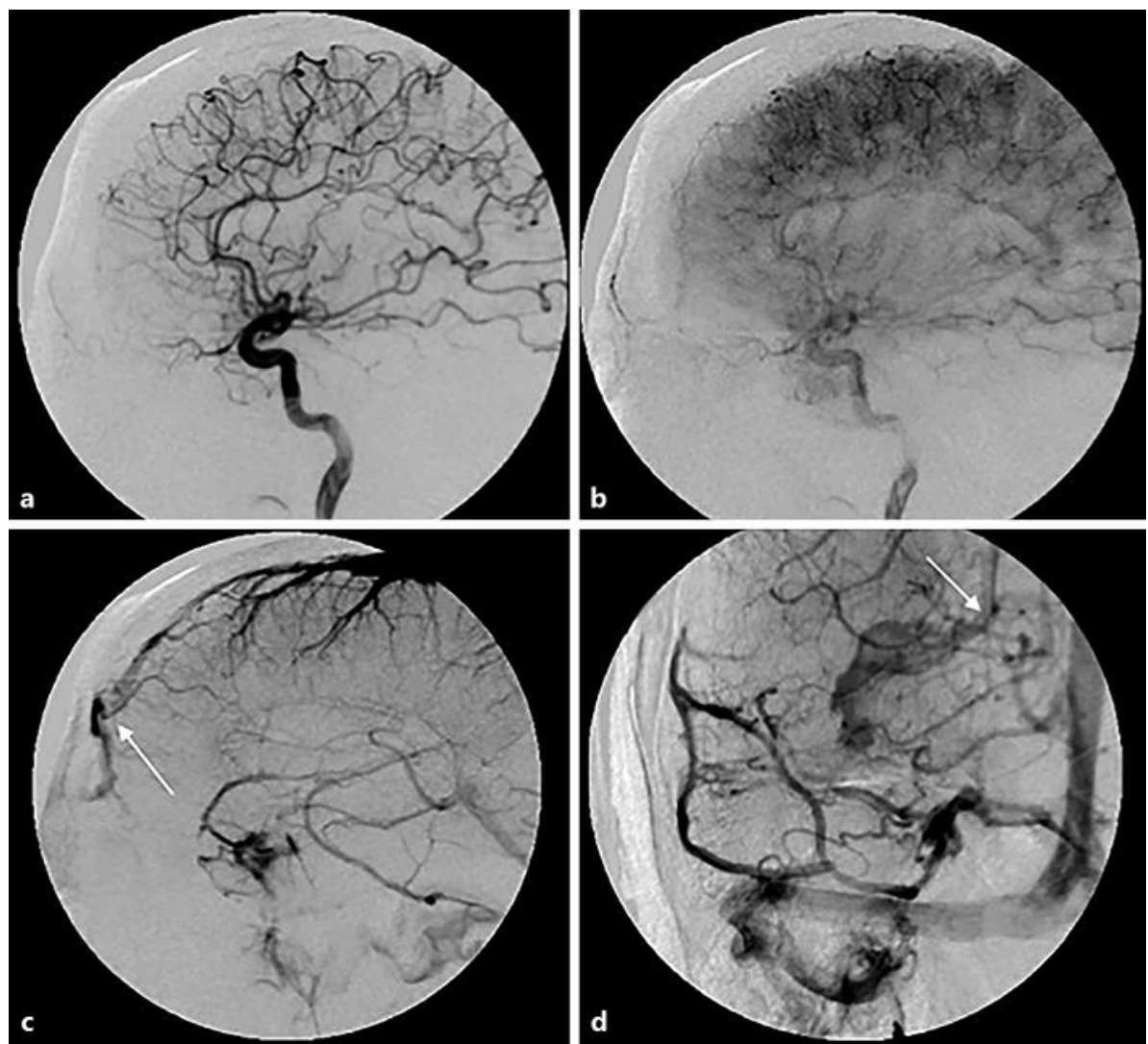

Fig. 3. Digital subtraction angiography arterial-phase (a) and capillary-phase (b) images reveal no vascular anomalies. Venous-phase images demonstrate a connection between the cerebral vein and an extracranial vein: lateral view (c), anteroposterior view (d). Each arrow indicates the connection between the venous lake and cortical vein. 